\title{
Energy spectrum of bound-spinons in the quantum Ising spin-chain ferromagnet
}

\author{
S.B. Rutkevich \\ December 6, 2007 \\ Institut für Theoretische Physik, Universität Münster, \\ Wilhelm-Klemm Str. 9, 48149 Münster, Germany, \\ and \\ Institute of Physics of Solids and Semiconductors, \\ P. Brovka Str. 17, Minsk 220072, Belarus \\ e-mail: rut@ifttp.bas-net.by
}

\begin{abstract}
We study the excitation energy spectrum in the $S=1 / 2$ ferromagnetic Ising spin chain with the easy axis $z$ in a magnetic field $\mathbf{h}=\left\{h_{x}, 0, h_{z}\right\}$. According to Wu and McCoy's scenario of weak confinement, the fermionic spinon excitations (kinks), being free at $h_{z}=0$ in the ordered phase, are coupled into bosonic bound states at arbitrary small $h_{z}>0$. We calculate the energy spectrum of such excitations in the leading order in small $h_{z}$, using different perturbative methods developed for the similar problem in the Ising field theory.
\end{abstract}

\section{Introduction}

In recent years much progress has been achieved in the understanding of various aspects of the scaling limit of the two-dimensional Ising model, which is known as the Ising Field Theory (IFT), for a review see [4]. It is described by the Euclidean action

$$
\mathcal{A}_{I F T}=\mathcal{A}_{C F T}+\tau \int \varepsilon(x) d^{2} x+h \int \sigma(x) d^{2} x .
$$


Here $\mathcal{A}_{I F T}$ corresponds to the conformal field theory in two dimensions, which is associated with the the Ising critical point. Fields $\varepsilon(x)$ (energy density) and $\sigma(x)$ (spin density) are the only relevant operators in the theory, their scaling dimensions are $X_{\epsilon}=1$ and $X_{\sigma}=1 / 8$. Parameters $\tau$ and $h$ are proportional to the deviation of the temperature $T$ and magnetic field $H$ in the lattice Ising model from their critical values: $\tau \sim T_{c}-T$ and $h \sim H$ at $T \rightarrow T_{c}, H \rightarrow 0$. In fact, only one dimensionless parameter $\eta=\tau /|h|^{8 / 15}$ determines the physics of IFT.

IFT is integrable along the directions $h=0$ and $\tau=0$. The former case $h=0, \tau \neq 0$ corresponds to Onsager's solution [16]. Exact solution of IFT at $\tau=0, h \neq 0$ was found by A.B. Zamolodchikov [23]. A thorough study of analytical properties of the free energy continued to complex values of the scaling parameter $\eta$ has been done by Fonseca and A.B. Zamolodchikov [10] by means of a numerical technique, known as the truncated free-fermion space approach [22]. Their analysis clarifies the relationship between the edge Yang-Lee singularity and the spinodal point.

For an analytical study of IFT for $h$ and $\tau$ close to the integrable directions, it is natural to exploit perturbation expansions. Form-factor perturbation theory developed by Delfino, Mussardo and Simonetti [6] has been applied to calculate the variation of the particle mass spectrum and the decay widths of non-stable particle for small $\eta$, i.e. near the line $\tau=0[5,6]$.

Perturbation theory for IFT in the region close to the $h=0$ axis turns out to be rather nontrivial in the low temperature phase $T<T_{c}$, as it was first shown by McCoy and $\mathrm{Wu}[14]$. At $h=0$ the particle sector of IFT contains one spinless fermion, which is interpreted in the ordered $T<T_{c}$ phase as a kink interpolating between two degenerate vacua. Application of magnetic field removes the degeneracy and induces a long-range interaction between kinks, which leads to their confinement into pairs. This means, that even at small field $h$ an isolated kink gains an infinite energy, and the bounded kink-antikink pairs become the only single-particle excitations in the model. Their dispersion law $E_{n}(P)$ has the relativistic form:

$$
E_{n}(P)=\sqrt{P^{2}+M_{n}^{2}}
$$

being completely determined by the masses $M_{n}$. This follows from the Lorentz invariance of IFT, which in turn is the result of the rotation invariance of the two-dimensional Ising model in the scaling limit.

At small $h$ the mass spectrum $M_{n}$ of kink-antikink pairs becomes dense in the segment $[2 m, \infty)$. Two asymptotic expansions describe $M_{n}$ at $h \rightarrow 0$ 
in different regions of this segment. Near the edge point $2 m$ (i.e. for fixed $n$ at $h \rightarrow 0$ ) one can use the low energy expansion in fractional powers of the magnetic field

$$
\frac{M_{n}^{2}}{4 m^{2}}=1+\sum_{k=2}^{\infty} \mu_{k} \zeta^{k / 3}
$$

where $m=2 \pi \tau$ is the mass of a free fermion (kink) at $h=0, \bar{\sigma}$ is the spontaneous magnetization at $h=0$, and $\zeta=2 h \bar{\sigma} / \mathrm{m}^{2}$. Several initial coefficients $\mu_{k}$ are known explicitly. The coefficient of the leading term has been found by McCoy and $\mathrm{Wu}[14]$ in the form $\mu_{2}=z_{n}$, where $-z_{n}$ denotes the zeroes of the Airy function, $\operatorname{Ai}\left(-z_{n}\right)=0$. Correction terms in (3) up to $k=8$ have been calculated by Fonseca and A.B. Zamolodchikov, see $[8,10]$.

On the other hand, for $n \gg 1$ and $h \rightarrow 0$ the semiclassical expansion in integer powers of $h$ can be applied:

$$
\frac{M_{n}^{2}}{4 m^{2}}=\left[1+a_{2} \zeta^{2}+O\left(\zeta^{3}\right)\right] \cosh \vartheta_{n}
$$

where [9] $a_{2}=0.071010809 \ldots$, and the numbers $\vartheta_{n}$ denote the solutions of equation

$$
\sinh 2 \vartheta_{n}-2 \vartheta_{n}=2 \pi \zeta(n-1 / 4)-\zeta^{2} \bar{S}_{1}\left(\vartheta_{n}\right)-O\left(\zeta^{3}\right),
$$

with

$$
\bar{S}_{1}(\vartheta)=-\frac{1}{\sinh 2 \vartheta}\left[\frac{5}{24} \frac{1}{\sinh ^{2} \vartheta}+\frac{1}{4} \frac{1}{\cosh ^{2} \vartheta}-\frac{1}{12}-\frac{1}{6} \sinh ^{2} \vartheta\right] .
$$

The leading terms of order $\zeta$ in (4), (5) were determined by Rutkevich [18], and independently by Fonseca and A.B. Zamolodchikov [8]. The second-order term (6) in (5) has been found by Fonseca and A.B. Zamolodchikov [8].

IFT viewed as a particle theory gives us a nice model of quark confinement. It is worth to note, that there are a lot of similarities between confinement in IFT and in 't Hooft's model for the two-dimensional multicolor QCD [11], see the discussion in [8]. On the other hand, IFT can be treated as the continuous model of the one-dimensional quantum ferro- (or antiferro-) magnet. The continuous approximation applies to one-dimensional ferromagnets only in the critical region near the quantum phase transition point [20]. Beyond the critical region the discreteness of the lattice become important. 
The purpose of the present paper is to discuss, how the picture of confinement outlined above is modified by the discrete lattice effects beyond the critical region. Preliminary results in this directions have been obtained in [19]. Our work is motivated by recent experimental observation of confinement of topological excitations in spin-1/2 chain antiferromagnet reported by Kenzelmann et al. [13]. Accordingly, we shall hold to the magnetic terminology: the terms "spinon", "domain wall" or "kink" will be used for topological excitations, and their bound-states will be called "bound-spinons" [13]. The problem will be considered within the simplest appropriate one-dimensional model of ferromagnetism: $S=1 / 2$ Ising spin chain in a "skew" magnetic field $[3,7]$, which has components both normal and parallel to the easy magnetization axis $z, \mathbf{h}=\left\{h_{x}, 0, h_{z}\right\}$. This model has an exact solution in the case of a purely transverse magnetic field $\mathbf{h}=\left\{h_{x}, 0,0\right\}$ and reduces to IFT in the critical region near the quantum phase transition point [20]. We focus our attention on the dispersion law of bound-spinons in the limit of small longitudinal magnetic field $h_{z} \rightarrow 0$, and extend the results (3), (44) to this model beyond the critical region 1 . It turns out, that the bound-spinon energy spectrum in the discrete spin chain exhibits a rather rich small- $h_{z}$ asymptotical behavior. We describe eight different regimes for the bound-spinon energy spectrum, which are realized in the noncritical Ising spin chain in the limit $h_{z} \rightarrow 0$, instead of two regimes (3), (4) known in IFT.

The rest of the paper is organized as follows. In Section 2 the spin$1 / 2$ Ising chain model in a skew magnetic field is described. We study the bound-spinon spectrum in this model by means of two different procedures, which are shown to be effective in the IFT $[8,18]$. In Section 3 we apply a heuristic approach based on the Bohr-Sommerfeld quantization rule. Results obtained in Section 3 are confirmed and extended in more systematic theory in subsequent sections. In Section 4 we derive a singular integral equation, which determines the energy spectrum of bound-spinons in the two-fermion approximation, and generalizes to the discrete spin chain model the IFT Bethe-Salpeter equation $[8,10]$. Section [5] contains some technical results relating to the singular integral equation (43). In Section 6 we derive exact solutions of two simplified versions of this equation, and give explicit expressions for the corresponding bound-spinon energy spectra. In Section 7 we return to the original integral equation (43), and develop for it a weak

\footnotetext{
${ }^{1}$ Of course, the relativistic functional form (2) does not hold for the bound-spinon spectrum in the discrete spin chain model apart from the critical region.
} 
coupling perturbative expansion based on the results of Section 5. The lowest order of this expansion leads to eight different small- $h_{z}$ asymptotics for the bound-spinon dispersion law, which can be used in different regions of the bound spinon quasimomentum and energy. Obtained results are discussed in Section 8.

\section{Ising model in skew magnetic field}

The quantum Ising spin-1/2 chain model is defined by the Hamiltonian:

$$
\mathcal{H}=-\sum_{j}\left(\sigma_{j}^{z} \sigma_{j+1}^{z}+h_{x} \sigma_{j}^{x}+h_{z} \sigma_{j}^{z}\right)
$$

Here $\sigma^{x, z}$ are the Pauli matrices, $j$ enumerates the chain sites. The number of the chain sites is put to infinity in the thermodynamical limit. The applied "skew" magnetic field $\mathbf{h}$ has both components parallel and normal to the magnetic easy axis $z$. The Hamiltonian is normalized to the ferromagnetic nearest neighbor coupling constant.

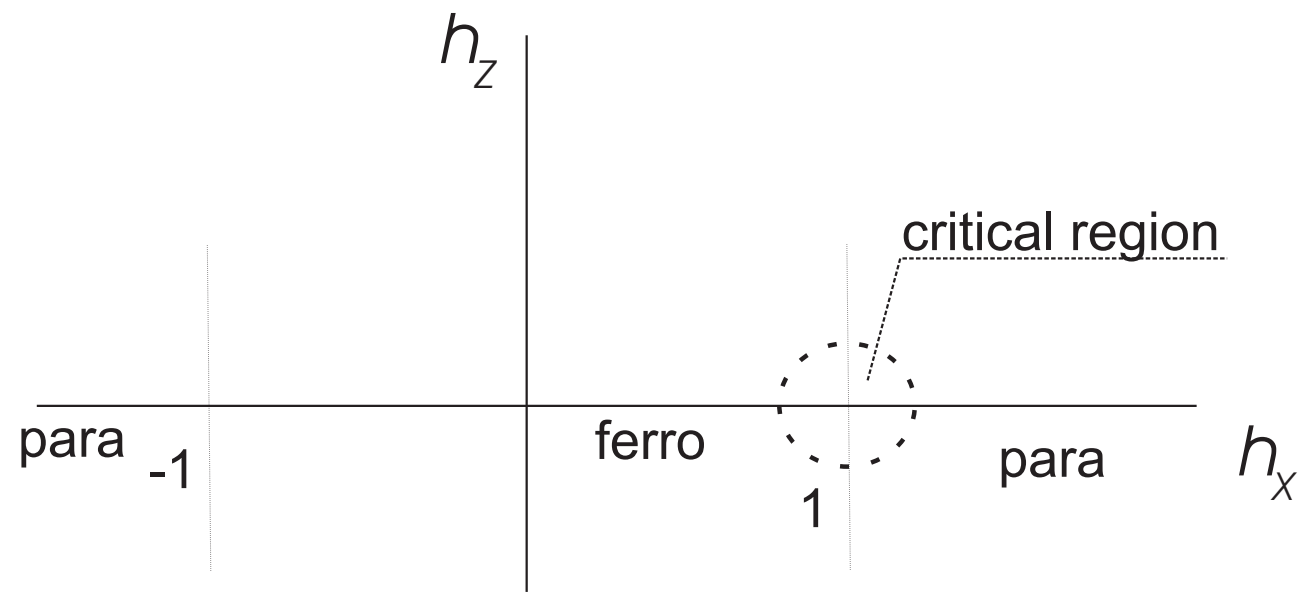

Figure 1: Phase diagram of the Ising spin chain at $T=0$.

The phase diagram of model (77) at zero temperature is shown in Fig. 1. Model (77) is not integrable at generic nonzero $h_{x}, h_{z}$. However, in the line $h_{z}=0$ it has exact solution first found by Pikin and Tsukernik [17]. At $h_{x}=$ \pm 1 lie the quantum phase transition points, which separate ferromagnetic 
and paramagnetic phases [20]. In the critical regions near these points the model is equivalent to IFT. Two ferromagnetic ground states $\left|0_{+}\right\rangle$and $\left|0_{-}\right\rangle$ coexist in the ferromagnetic phase $-1<h_{x}<1$. These ground states have the same energies $E_{+}\left(h_{z}=0\right)=E_{-}\left(h_{z}=0\right)$, but the opposite signs of the spontaneous magnetization $\left\langle 0_{ \pm}\left|\sigma_{j}^{z}\right| 0_{ \pm}\right\rangle= \pm \bar{\sigma}$, where $\bar{\sigma}=\left(1-h_{x}^{2}\right)^{1 / 8}$.

In the integrable line $h_{z}=0$ the model Hamiltonian (17) can be reduced to free fermions by use of the Jordan-Wigner transformation [12,17]:

$$
\left.\mathcal{H}_{0} \equiv \mathcal{H}_{c h}\right|_{h_{z=0}}=\int_{-\pi}^{\pi} \frac{\mathrm{d} \theta}{2 \pi} \omega(\theta) a^{\dagger}(\theta) a(\theta)+\text { const }
$$

where $\theta$ is the quasi-momentum, fermionic operators $a^{\dagger}(\theta), a(\theta)$ satisfy the canonical anticommutation relations

$$
\begin{aligned}
\left\{a(\theta), a\left(\theta^{\prime}\right)\right\} & =\left\{a^{\dagger}(\theta), a^{\dagger}\left(\theta^{\prime}\right)\right\}=0, \\
\left\{a^{\dagger}(\theta), a\left(\theta^{\prime}\right)\right\} & =2 \pi \delta\left(\theta-\theta^{\prime}\right),
\end{aligned}
$$

and the free-fermion dispersion law is given by

$$
\omega(\theta)=2\left[\left(1-h_{x}\right)^{2}+4 h_{x} \sin ^{2} \frac{\theta}{2}\right]^{1 / 2} .
$$

Operators $\sigma_{j}^{x}$ and $\sigma_{j}^{z} \sigma_{j+1}^{z}$ are bilinear in fermionic fields. Operator $\sigma_{j}^{z}$ can be written as a normally ordered exponential of a bilinear form of fermionic operators [12]. It can be also completely characterized by the form-factors:

$$
\left\langle\theta_{1}, \ldots, \theta_{K}\left|\sigma_{0}^{z}\right| \theta_{1}^{\prime} \ldots, \theta_{N}^{\prime}\right\rangle=\left\langle 0\left|a\left(\theta_{1}\right), \ldots, a\left(\theta_{K}\right) \sigma_{0}^{z} a^{\dagger}\left(\theta_{1}^{\prime}\right), \ldots, a^{\dagger}\left(\theta_{N}^{\prime}\right)\right| 0\right\rangle,
$$

where $(K+N)$ takes even values. The elementary form-factors are given by:

$$
\begin{array}{r}
\bar{\sigma}^{-1}\left\langle\theta_{1}, \theta_{2}\left|\sigma_{0}^{z}\right| 0\right\rangle=F\left(\theta_{1}, \theta_{2} \mid\right)=\frac{1}{1-\exp \left[i\left(\theta_{1}+\theta_{2}\right)\right]} \frac{\omega\left(\theta_{1}\right)-\omega\left(\theta_{2}\right)}{\sqrt{\omega\left(\theta_{1}\right) \omega\left(\theta_{2}\right)}}, \\
\bar{\sigma}^{-1}\left\langle 0\left|\sigma_{0}^{z}\right| \theta_{1}, \theta_{2}\right\rangle=F\left(\mid \theta_{1}, \theta_{2}\right)=\frac{1}{\exp \left[-i\left(\theta_{1}+\theta_{2}\right)\right]-1} \frac{\omega\left(\theta_{1}\right)-\omega\left(\theta_{2}\right)}{\sqrt{\omega\left(\theta_{1}\right) \omega\left(\theta_{2}\right)}}, \\
\bar{\sigma}^{-1}\left\langle\theta\left|\sigma_{0}^{z}\right| \theta^{\prime}\right\rangle=F\left(\theta \mid \theta^{\prime}\right)=\frac{1}{1-\exp \left[i\left(\theta-\theta^{\prime}\right)\right]} \frac{\omega(\theta)+\omega\left(\theta^{\prime}\right)}{\sqrt{\omega(\theta) \omega\left(\theta^{\prime}\right)}} .
\end{array}
$$

All other form-factors can be obtained from the elementary ones by application of the Wick rule. For example, the form-factor with two fermions in the initial and final states can be written as:

$$
\frac{\left\langle\theta_{1}, \theta_{2}\left|\sigma_{0}^{z}\right| \theta_{1}^{\prime} \theta_{2}^{\prime}\right\rangle}{\bar{\sigma}}=F\left(\theta_{1}, \theta_{2} \mid\right) F\left(\mid \theta_{1}^{\prime}, \theta_{2}^{\prime}\right)+F\left(\theta_{1} \mid \theta_{2}^{\prime}\right) F\left(\theta_{2} \mid \theta_{1}^{\prime}\right)-F\left(\theta_{1} \mid \theta_{1}^{\prime}\right) F\left(\theta_{2} \mid \theta_{1}^{\prime}\right) \text {. }
$$




\section{Bound states of two domain walls}

In the ferromagnetic phase $\left|h_{x}\right|<1$, the free fermions in (7) represent the domain walls, which separate regions with different orientations of the magnetization. A small longitudinal magnetic field $h_{z}>0$, which breaks the $\mathbb{Z}_{2}$-symmetry, evidently provides the long range attraction force $\chi=$ $2 h_{z} \bar{\sigma}$ between the two neighboring domain walls. Due to this confining force, all domain walls become coupled into pairs which we shall call boundspinons, following [13]. If $h_{z}$ is small, the weak confinement regime is realized. The energy spectrum of bound-spinons in this regime can be understood to much extent in the following heuristic approach, first developed for the IFT $[8,10,14,18]$.

Consider two interacting fermions moving in a line as a classical system with the Hamiltonian

$$
\mathcal{H}\left(\theta_{1}, \theta_{2}, x_{1}, x_{2}\right)=\omega\left(\theta_{1}\right)+\omega\left(\theta_{2}\right)+\chi\left|x_{2}-x_{1}\right|
$$

Here the fermion coordinates $x_{1}, x_{2}$ are the real numbers. Variables $\theta_{1}, \theta_{2}$ are the canonical momenta corresponding to the coordinates $x_{1}, x_{2}$, and the fermion kinetic energy $\omega(\theta)$ is given by (9). After the canonical transformation

$$
\begin{aligned}
& X=\frac{x_{1}+x_{2}}{2}, x=x_{2}-x_{1}, \\
& \Theta=\theta_{1}+\theta_{2}, \theta=\frac{\theta_{2}-\theta_{1}}{2},
\end{aligned}
$$

the Hamiltonian (14) takes the form

$$
\mathcal{H}(\theta, x ; \Theta)=\epsilon(\theta ; \Theta)+\chi|x|,
$$

where

$$
\epsilon(\theta ; \Theta)=\omega(\theta+\Theta / 2)+\omega(\theta-\Theta / 2) .
$$

The total energy-momentum conservation laws read as:

$$
\begin{aligned}
& \epsilon(\theta(t) ; \Theta)+\chi|x(t)|=E=\text { Const, } \\
& \Theta(t)=\text { Const. }
\end{aligned}
$$




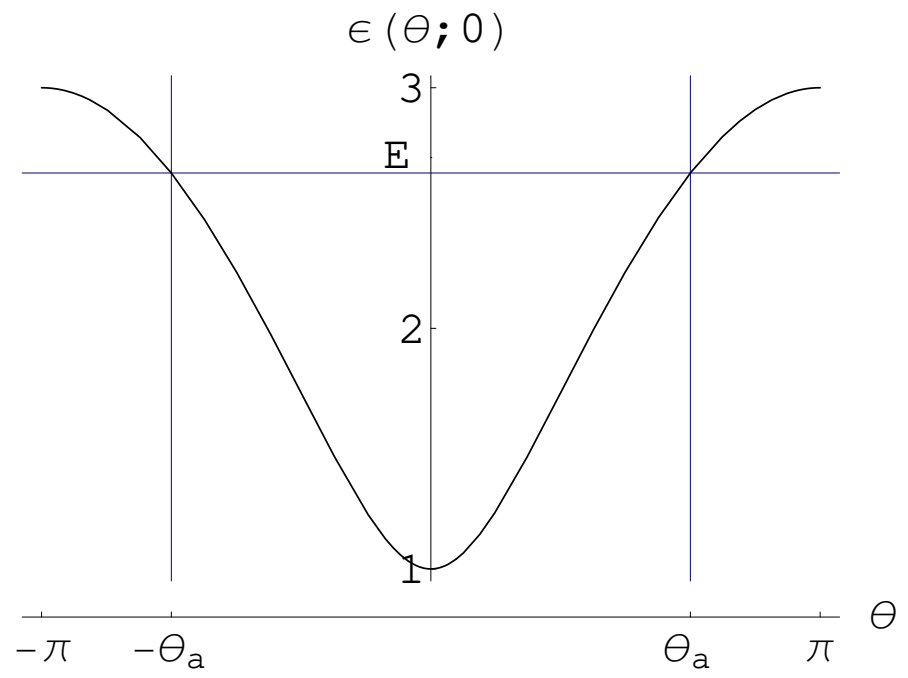

Figure 2: Function $\epsilon(\theta ; \Theta)$ given by (17), (9) for $\Theta=0$ and $h_{x}=0.5$. For energy $E$ classically allowed region is $\left[-\theta_{a}, \theta_{a}\right]$.

The canonical equations of motion are:

$$
\begin{aligned}
& \dot{X}(t)=\frac{\partial \epsilon(\theta ; \Theta)}{\partial \Theta}, \\
& \dot{x}(t)=\frac{\partial \epsilon(\theta ; \Theta)}{\partial \theta}, \\
& \dot{\theta}(t)=-\chi \operatorname{sign}[x(t)] .
\end{aligned}
$$

For a given value of the conserved total momentum $\Theta$, Hamiltonian (16) describes the relative motion of two fermions. This motion becomes especially simple in the $\theta$-space.

Fig. 2 shoes the $\theta$-dependence of the "kinetic energy" $\epsilon(\theta ; \Theta)$ for $h_{x}=0.5$ and $\Theta=0$. If $\theta=\theta_{a}$ and $x=+0$ at $t=0$, the total energy takes the value $E=\epsilon\left(\theta_{a}, \Theta\right)$. Due to (21), the momentum $\theta$ of the relative motion will linearly decreases in time

$$
\theta(t)=\theta_{a}-\chi t
$$

until the moment $t=t_{1}=2 \theta_{a} / \chi$, when $\theta(t)$ reaches the value $-\theta_{a}$. The time variation of the space coordinate $x(t)$ for $0<t<t_{1}$ can be read from (18)):

$$
x(t)=\frac{E-\epsilon[\theta(t) ; \Theta]}{\chi} .
$$


Then $x(t)$ becomes negative, and in the interval $t_{1}<t<2 t_{1}$ canonical coordinates vary as

$$
\begin{aligned}
& \theta(t)=-\theta_{a}+\left(t-t_{1}\right) \chi, \\
& x(t)=-\frac{E-\epsilon[\theta(t) ; \Theta]}{\chi} .
\end{aligned}
$$

Then the phase trajectories periodically repeat in time with the period $2 t_{1}$. The semiclassical energy levels can be obtained from the Bohr-Sommerfeld quantization condition:

$$
\oint d x \theta=2 \pi(\nu+1 / 2)
$$

where integration is taken along the closed periodic phase path in the $(x, \theta)$ plane over one cycle of motion, $0<t<2 t_{1}$. Since the two interacting particles are fermions, only odd values are allowed for the integer $\nu$ due to the Pauli principle:

$$
\nu=2 n-1, \quad n=1,2, \ldots .
$$

The right-hand side of (24) can be transformed as

$$
\begin{gathered}
\oint d x \theta=-2 \int_{-\theta_{a}}^{\theta_{a}} d \theta \theta \frac{d x(\theta)}{d \theta}=\frac{2}{\chi} \int_{-\theta_{a}}^{\theta_{a}} d \theta \theta \dot{x}(t)= \\
\frac{2}{\chi} \int_{-\theta_{a}}^{\theta_{a}} d \theta \theta \frac{\partial \epsilon(\theta ; \Theta)}{\partial \theta}=\frac{2}{\chi}\left(2 E \theta_{a}-\int_{-\theta_{a}}^{\theta_{a}} d \theta \epsilon(\theta ; \Theta)\right) .
\end{gathered}
$$

Here in the second equality we have taken into account the linear dependence (22) between $\theta$ and $t$ for $0<t<t_{1}$, and in the third equality we have used equation of motion (20). Combining (24), (25), and (26) we find the semiclassical energy spectrum $E_{n}(\Theta)$ of bound states in the system of two fermions (14):

$$
2 E_{n}(\Theta) \theta_{a}-\int_{-\theta_{a}}^{\theta_{a}} d \theta \epsilon(\theta, \Theta)=2 \pi \chi(n-1 / 4), \quad n=1,2, \ldots
$$

where $\theta_{a} \in[0, \pi]$ is the solution of equation

$$
\epsilon\left(\theta_{a} ; \Theta\right)=E_{n}(\Theta)
$$


In the above treatment we implied that function $\epsilon(\theta ; \Theta)$ monotonically increases with $\theta$ in the interval $0<\theta<\pi$, providing that equation (28) has a single solution there for $E \in[\epsilon(0 ; \Theta), \epsilon(\pi ; \Theta)]$. This is true for small enough values of the total momentum $\Theta$ :

$$
0<\Theta<\Theta_{m}, \quad \Theta_{m}=2 \arccos h_{x} .
$$

However, for $\Theta>\Theta_{m}$ the second derivative $\partial^{2} \epsilon(\theta ; \Theta) / \partial \theta^{2}$ at $\theta=0$ becomes negative, see Fig. 3. Though for high enough energies $\epsilon(0, \Theta)<E<\epsilon(\pi, \Theta)$ we can still use formula (27), for smaller energies $E<\epsilon(0, \Theta)$, equation (18) has two solutions $\theta_{a}, \theta_{b}$ in the interval $0<\theta<\pi$. In the latter case the classical phase trajectory $\theta(t)$ moves back and forth in one of the the classically allowed region, say in the interval $\left[\theta_{b}, \theta_{a}\right]$. Equations (22), (23) remain still valid, with $t_{1}=\left(\theta_{a}-\theta_{b}\right) / \chi$, however. Semiclassical energy levels

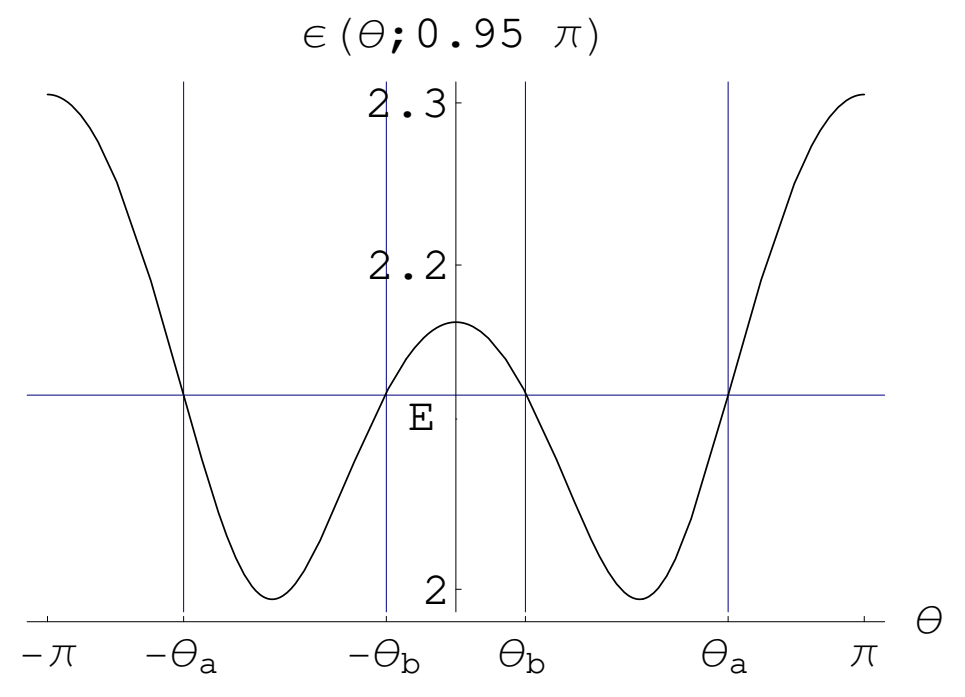

Figure 3: Function $\epsilon(\theta ; \Theta)$ determined by (17) (9) for $\Theta=0.95 \pi$ and $h_{x}=$ 0.5 . For energy $E$ classically allowed regions are $\left[-\theta_{a},-\theta_{b}\right]$ and $\left[\theta_{b}, \theta_{a}\right]$.

are determined by condition (24), where the integration path is located now in the interval $\left[\theta_{b}, \theta_{a}\right]$. Since this interval is not invariant under reflection $\theta \rightarrow-\theta$, the Pauli principle does not impose any restrictions on the integers $\nu=0,1,2, \ldots$ in (24). Therefore, instead of (27), we get for $\Theta_{m}<\Theta<\pi$ and $E<\epsilon(0, \Theta)$ :

$$
E_{n}(\Theta)\left(\theta_{a}-\theta_{b}\right)-\int_{\theta_{b}}^{\theta_{a}} d \theta \epsilon(\theta, \Theta)=\pi \chi(n-1 / 2), \quad n=1,2, \ldots,
$$


where $n=\nu+1$, and $\theta_{a}, \theta_{b} \in[0, \pi]$ are the solutions of equation

$$
\epsilon\left(\theta_{a, b}, \Theta\right)=E_{n}(\Theta),
$$

shown in Fig. 3 .

Semiclassical formulas (27), (29) can be used, if $n$ is large, $n \gg 1$, and the energy $E_{n}(\Theta)$ does not approach one of the critical values $E_{c}(\Theta)$ of the function $\epsilon(\theta, \Theta): \quad E_{c}(\Theta)=\epsilon\left(\theta_{c}, \Theta\right), \quad \partial \epsilon(\theta, \Theta) /\left.\partial \theta\right|_{\theta=\theta_{c}}=0$. On the other hand, the low-energy part of the spectrum for small $h_{z} \rightarrow 0$ and $0<\Theta<\Theta_{m}$ can be determined by use of the "nonrelativistic approximation" developed for the IFT $[8,10,14]$. One should expand the "kinetic energy" in $\theta$ near the origin $\epsilon(\theta, \Theta) \approx \varepsilon_{0}(\Theta)+\varepsilon_{2}(\Theta) \theta^{2} / 2$ reducing the problem to the Schrödinger equation with the Hamiltonian $\varepsilon_{0}(\Theta)-\frac{1}{2} \varepsilon_{2}(\Theta) \partial_{x}^{2}+\chi|x|$. Only odd wave eigenfunctions $\phi(x)$ should be taken into account due to the Pauli principle, and the energy levels take the form

$$
E_{n}(\Theta) \approx \varepsilon_{0}(\Theta)+\chi^{2 / 3}\left[\varepsilon_{2}(\Theta) / 2\right]^{1 / 3} z_{n}
$$

where

$$
\varepsilon_{0}(\Theta)=\epsilon(0, \Theta), \quad \varepsilon_{2}(\Theta)=\partial^{2} \epsilon(\theta, \Theta) /\left.\partial \theta^{2}\right|_{\theta=0},
$$

and $-z_{n}, n=1,2, \ldots$ are the zeros of the Airy function $\operatorname{Ai}\left(-z_{n}\right)=0$.

\section{Two-fermion approximation in the Ising chain model}

The bound-spinon energy spectrum can be formally defined as the solution of the eigenvalue problem

$$
\left(\mathcal{H}-E_{\mathrm{vac}}\right)\left|\Phi_{n}(\Theta)\right\rangle=E_{n}(\Theta)\left|\Phi_{n}(\Theta)\right\rangle,
$$

where $\mathcal{H}$ is the Ising chain Hamiltonian (17), $E_{\text {vac }}$ is the ground state energy, and $\left|\Phi_{n}(\Theta)\right\rangle$ is the bound-spinon eigenvector with quasimomentum $\Theta$ : $\hat{T}_{1}\left|\Phi_{n}(\Theta)\right\rangle=\exp (i \Theta)\left|\Phi_{n}(\Theta)\right\rangle$, with $\hat{T}_{1}$ being the lattice site translation operator. In the small- $h_{z}$ limit this problem can be studied within the two-fermion approximation, which turns out to be effective in IFT $[8,10,18]$. In this approach one considers instead of the the exact eigenvalue problem (31) its projection to the two-fermion subspace $\mathcal{F}_{2}$ of the Fock space

$$
\mathcal{P}_{2} \mathcal{H} \mathcal{P}_{2}\left|\Phi_{n}(\Theta)\right\rangle=E_{n}(\Theta) \mathcal{P}_{2}\left|\Phi_{n}(\Theta)\right\rangle
$$


where $\mathcal{P}_{2}$ is the orthogonal projector onto $\mathcal{F}_{2}$. So, the bound-spinon state is approximated by a two-fermion state, neglecting the four-fermion, six fermion, and higher multi-fermion contributions. In the momentum representation (34) reads as

$$
\begin{array}{r}
{\left[\omega\left(\theta_{1}\right)+\omega\left(\theta_{1}\right)-E_{n}(\Theta)\right] \phi_{n}\left(\theta_{1}, \theta_{2}\right)=\frac{h_{z}}{2} \iint_{-\pi}^{\pi} \frac{d \theta_{1}^{\prime} d \theta_{2}^{\prime}}{2 \pi} \phi_{n}\left(\theta_{1}^{\prime}, \theta_{2}^{\prime}\right) .} \\
\sum_{k} \delta\left(\theta_{1}^{\prime}+\theta_{2}^{\prime}+2 \pi k-\Theta\right)\left\langle\theta_{2}, \theta_{1}\left|\sigma_{0}^{z}\right| \theta_{1}^{\prime} \theta_{2}^{\prime}\right\rangle,
\end{array}
$$

where

$$
\begin{array}{r}
\phi_{n}\left(\theta_{1}, \theta_{2}\right)=\left\langle\theta_{2}, \theta_{1}\left|\mathcal{P}_{2}\right| \Phi_{n}(\Theta)\right\rangle, \\
\phi_{n}\left(\theta_{2}, \theta_{1}\right)=-\phi_{n}\left(\theta_{1}, \theta_{2}\right), \\
\theta_{1}+\theta_{2}=\Theta+2 \pi k, \quad k=0, \pm 1, \pm 2 \ldots
\end{array}
$$

Taking into account (13) and (36), we can replace the two-fermion form-factor in the integrand, as

$$
\begin{aligned}
&\left\langle\theta_{2}, \theta_{1}\left|\sigma_{0}^{z}\right| \theta_{1}^{\prime} \theta_{2}^{\prime}\right\rangle \rightarrow 4 \bar{\sigma} \mathcal{G}\left(\theta_{2}, \theta_{1} \mid \theta_{1}^{\prime}, \theta_{2}^{\prime}\right), \\
& \mathcal{G}\left(\theta_{2}, \theta_{1} \mid \theta_{1}^{\prime}, \theta_{2}^{\prime}\right)=\frac{1}{4}\left[F\left(\theta_{2}, \theta_{1} \mid\right) F\left(\mid \theta_{1}^{\prime}, \theta_{2}^{\prime}\right)+2 F\left(\theta_{1} \mid \theta_{1}^{\prime}\right) F\left(\theta_{2} \mid \theta_{2}^{\prime}\right)\right],
\end{aligned}
$$

where the elementary form-factors $F(.$.$) are given by (10). Since the bound-$ spinon quasimomentum $\Theta$ is fixed, one can rewrite (35) in the variables $\theta=\theta_{2}-\theta_{1}$ and $\theta^{\prime}=\theta_{2}^{\prime}-\theta_{1}^{\prime}$ :

$$
\left[\epsilon(\theta ; \Theta)-E_{n}(\Theta)\right] \phi_{n, \Theta}(\theta)=\chi \int_{-\pi}^{\pi} \frac{d \theta^{\prime}}{2 \pi} G_{\Theta}\left(\theta, \theta^{\prime}\right) \phi_{n, \Theta}\left(\theta^{\prime}\right)
$$

where $\epsilon(\theta ; \Theta)$ is given by (17), $\chi=2 h_{z} \bar{\sigma}$, and

$$
G_{\Theta}\left(\theta, \theta^{\prime}\right)=\mathcal{G}\left(\frac{\Theta}{2}+\theta, \frac{\Theta}{2}-\theta \mid \frac{\Theta}{2}-\theta^{\prime}, \frac{\Theta}{2}+\theta^{\prime},\right)
$$

The kernel has the second order pole singularity at $\theta=\theta^{\prime}$ :

$$
G_{\Theta}\left(\theta, \theta^{\prime}\right)=-\frac{2}{\left\{\exp \left[i\left(\theta-\theta^{\prime}\right) / 2\right]-\exp \left[-i\left(\theta-\theta^{\prime}\right) / 2\right]\right\}^{2}}+G_{\Theta}^{(r e g)}\left(\theta, \theta^{\prime}\right),
$$


where $G_{\Theta}^{(r e g)}\left(\theta, \theta^{\prime}\right)$ is regular at real $\theta$ and $\theta^{\prime}$.

The function $\phi_{n, \Theta}(\theta)$ in equation (37) is odd and $2 \pi$-periodic in $\theta$, and the integral in the right-hand side is understood in the sense of the Cauchy principal value. Equation (37) gives the discrete-lattice version of the IFT Bethe-Salpeter equation in a generic momentum frame, see (3.11) in [8]. It is instructive to rewrite (37) in the coordinate representation:

$$
\begin{array}{r}
\sum_{j^{\prime}} K\left(j-j^{\prime}\right) \phi_{n, \Theta}\left(j^{\prime}\right)-E_{n}(\Theta) \phi_{n, \Theta}(j)= \\
-\chi|j| \phi_{n, \Theta}(j)+\chi \sum_{j^{\prime}} \mathcal{U}\left(j, j^{\prime}\right) \phi_{n, \Theta}\left(j^{\prime}\right),
\end{array}
$$

where $K(j)$ and $\phi_{n, \Theta}(j)$ are the Fourier coefficients of the $2 \pi$-periodic functions $\epsilon(\theta ; \Theta)$ and $\phi_{n, \Theta}(\theta)$, respectively, $j, j^{\prime}=0, \pm 1, \ldots$

The first and the second terms in the right-hand side of (40) come, respectively, from the first and the second terms in (39). The first term describes the long-range attraction between fermions (domain walls), while the second, being responsible for the short-range interaction, vanishes exponentially in $|j|$ and $\left|j^{\prime}\right|$ on the correlation length scale.

In this paper we shall focus our attention on the perturbative solution of the singular integral equation (37) in the leading order in the small parameter $\chi$. As we know from the IFT, the short-range interaction does not effect the fermion bound-state energy in the leading order in $h_{z}$, contributing only to the higher order corrections $[8,18]$. Accordingly, in what follows we shall drop the second term in the right-hand side of (39), which does not contribute to the bound-spinon energy $E_{n}(\Theta)$ in the leading order in $\chi$. Then, equation (37) takes the form in variables $z=\exp (i \theta), z^{\prime}=\exp \left(i \theta^{\prime}\right)$ :

$$
[\epsilon(z ; \Theta)-E(\Theta)] \phi(z)=-\chi z f_{S_{1}} \frac{d z^{\prime}}{\pi i} \frac{\phi\left(z^{\prime}\right)}{\left(z^{\prime}-z\right)^{2}},
$$

where

$$
\epsilon(z ; \Theta)=2 \sqrt{h_{x}}\left\{\left[h_{x}+\frac{1}{h_{x}}-z v-\frac{1}{z v}\right]^{1 / 2}+\left[h_{x}+\frac{1}{h_{x}}-\frac{z}{v}-\frac{v}{z}\right]^{1 / 2}\right\}
$$

and $v=\exp (i \Theta / 2)$. We use notation $S_{r}$ for the circle of the radius $r$ centered in the origin and passed in the counter-clockwise direction, and skip indices at $E_{n}(\Theta)$ and $\phi_{n, \Theta}(z)$. 


\section{Singular integral equation in the unit circle}

In the present Section we describe some general properties of equation (41), which will be used in Section 6 and 7 .

Consider a more general singular integral equation 2

$$
[\epsilon(z)-\lambda] \phi(z)=-\mu z f_{S_{1}} \frac{d z^{\prime}}{\pi i} \frac{\phi\left(z^{\prime}\right)}{\left(z^{\prime}-z\right)^{2}}
$$

Functions $\epsilon(z)$ and $\phi(z)$ are supposed to be analytical in a narrow ring $\Gamma$, $\Gamma=\{z \in \Gamma|1-\delta<| z \mid<1+\delta\}$, with small positive $\delta$. The symmetry properties $\epsilon(1 / z)=\epsilon(z)$ and $\phi(1 / z)=-\phi(z)$ are also required.

Let us define two functions $g_{+}(z)$ and $g_{-}(z)$ :

$$
g_{ \pm}(z)=\oint_{S_{1}} \frac{d z^{\prime}}{2 \pi i} \frac{\phi\left(z^{\prime}\right)}{\left(z^{\prime}-z\right)}
$$

where $g_{+}(z)$ is defined at $|z|<1$, and $g_{-}(z)$ is defined in the region $|z|>1$. The evident properties of these function are:

1. $g_{+}(z)$ and $g_{-}(z)$ are analytical at $|z|<1$ and at $|z|>1$, respectively.

2. $g_{+}(z)$ and $g_{-}(z)$ can be continued to the unit circle $S_{1}$, where they are continuous together with their derivatives.

3. $g_{+}(1 / z)=g_{-}(z)$.

4. $g_{+}(0)=g_{-}(\infty)=0$.

5. $g_{+}(z)-g_{-}(z)=\phi(z)$ for $|z|=1$.

6. $\partial_{z} g_{+}(z)+\partial_{z} g_{-}(z)=(\pi i)^{-1} f_{S_{1}} d z^{\prime} \phi\left(z^{\prime}\right)\left(z^{\prime}-z\right)^{-2}$ for $|z|=1$.

Combining properties 5, 6] with (43), one finds for $|z|=1$

$$
\left\{[\epsilon(z)-\lambda]+\mu z \partial_{z}\right\} g_{+}(z)=U(z)=\left\{[\epsilon(z)-\lambda]-\mu z \partial_{z}\right\} g_{-}(z) .
$$

The function $U(z)$ defined this way is invariant with respect to the inversion

$$
U(1 / z)=U(z),
$$

\footnotetext{
${ }^{2}$ On the general theory of singular integral equations see [15].
} 
and can be analytically continued from the circle $S_{1}$ into its neighborhood $\Gamma$. $U(z)$ can be expressed directly in terms of function $\phi(z)$ :

$$
U(z)=\int_{S_{1}} \frac{d z^{\prime}}{2 \pi i} \phi\left(z^{\prime}\right) \frac{\epsilon(z)-\epsilon\left(z^{\prime}\right)}{z^{\prime}-z} .
$$

Note, that the integrand in (47) is regular at $z^{\prime} \rightarrow z$.

Let us prove this useful relation, following ideas of Vekua [21]. We start from the Cauchy formula

$$
U(z)=\int_{\partial \Gamma} \frac{d t}{2 \pi i} \frac{U(t)}{t-z},
$$

where $|z|=1$, and $\partial \Gamma=S_{1+\delta}-S_{1-\delta}, S_{1 \pm \delta}$ denote circles of radius $1 \pm \delta$ passed counter clockwise. Substitution of (45) into (48) yields

$$
\begin{aligned}
U(z)= & \int_{S_{1+\delta}} \frac{d t}{2 \pi i} \frac{[\epsilon(t)-\lambda] g_{-}(t)-\mu t g_{-}^{\prime}(t)}{t-z}- \\
& \int_{S_{1-\delta}} \frac{d t}{2 \pi i} \frac{[\epsilon(t)-\lambda] g_{+}(t)+\mu t g_{+}^{\prime}(t)}{t-z} .
\end{aligned}
$$

Several terms vanish in (49) after integration, since $g_{-}(t)$ is analytical at $|t|>1$, and $g_{+}(t)$ is analytical at $|t|<1$. After substitution of (44) in (49), the result reads as

$$
U(z)=\int_{\partial \Gamma} \frac{d t}{2 \pi i} \frac{\epsilon(t)}{t-z} \int_{S_{1}} \frac{d z^{\prime}}{2 \pi i} \frac{\phi\left(z^{\prime}\right)}{z^{\prime}-t} .
$$

Change of the order of integration leads to (47), since

$$
\int_{\partial \Gamma} \frac{d t}{2 \pi i} \frac{\epsilon(t)}{(t-z)\left(z^{\prime}-t\right)}=\frac{\epsilon(z)-\epsilon\left(z^{\prime}\right)}{z^{\prime}-z}
$$

for $z, z^{\prime} \in S_{1}$.

It is helpful to inverse relation (45) and to express $g_{+}(z)$ in terms of $U(z)$ :

$$
g_{+}(z)=\int_{z_{0}}^{z} \frac{d z^{\prime}}{\mu z^{\prime}} U\left(z^{\prime}\right) \exp \left\{\frac{i}{\mu}\left[\mathcal{F}\left(z^{\prime}\right)-\mathcal{F}(z)\right]\right\},
$$

where

$$
i \mathcal{F}(z)=\int_{1}^{z} \frac{d t}{t}[\epsilon(t)-\lambda]
$$


The choice of the integration path and its initial point $z_{0}$ in (50) depends on $\epsilon(z)$, as will be discussed later. The general relations described here will be used in subsequent sections in analysis of equation (43) for particular choices of function $\epsilon(z)$. In Section 6 we shall describe two exact solutions of (43) for rational $\epsilon(z)$. In Section 7 we perform a perturbative analysis in the limit $\mu \rightarrow 0$ of equation (43) with $\epsilon(z)$ given by (42).

\section{Toy models}

Equation (43) can be solved exactly, if $\epsilon(z)$ is rational. In this case $U(z)$ is also rational, as follows immediately from (45). Furthermore, poles of $U(z)$ in the complex $z$-plane can be located only at the poles of $\epsilon(z)$. This information is sufficient to obtain the explicit solution of equation (43). In the present Section we describe this procedure in more detail for two rational $\epsilon(z)$ :

$$
\begin{aligned}
& \text { 1. } \epsilon(z)=\epsilon_{1}(z)=-\frac{1}{2}\left(z+z^{-1}\right), \\
& \text { 2. } \epsilon(z)=\epsilon_{2}(z)=-\left(z+z^{-1}\right)-\rho\left(z^{2}+z^{-2}\right) .
\end{aligned}
$$

The reason for studying such exactly solvable "toy models" is twofold. First, on the exact solutions one can check the accuracy of perturbative methods. Second, function (42), which arises in the Ising spin chain problem, can be approximated rather well by the function $\left[B_{0}+B_{1} \epsilon_{2}(z)\right]$ with certain constants $B_{0}, B_{1}$, if $h_{x}$ is not close to the quantum phase transition point.

\subsection{Toy model 1}

In this subsection we consider a toy model, in which free fermions at $h_{z}=0$ have the dispersion law $\omega(\theta)=-\cos \theta$. Then function (17) reduces to

$$
\epsilon(\theta ; \Theta)=-2 \cos (\Theta / 2) \cos \theta
$$

and equation (41) takes the form:

$$
\left[\left(-z-z^{-1}\right) \cos (\Theta / 2)-E(\Theta)\right] \phi(z)=-\chi z \int_{S_{1}} \frac{d z^{\prime}}{\pi i} \frac{\phi\left(z^{\prime}\right)}{\left(z^{\prime}-z\right)^{2}} .
$$

After rescaling

$$
\lambda=\frac{E(\Theta)}{2 \cos (\Theta / 2)}, \quad \mu=\frac{\chi}{2 \cos (\Theta / 2)},
$$


we come to equation (43) with $\epsilon(z)=\epsilon_{1}(z)$. It is clear from (45), that $U(z)$

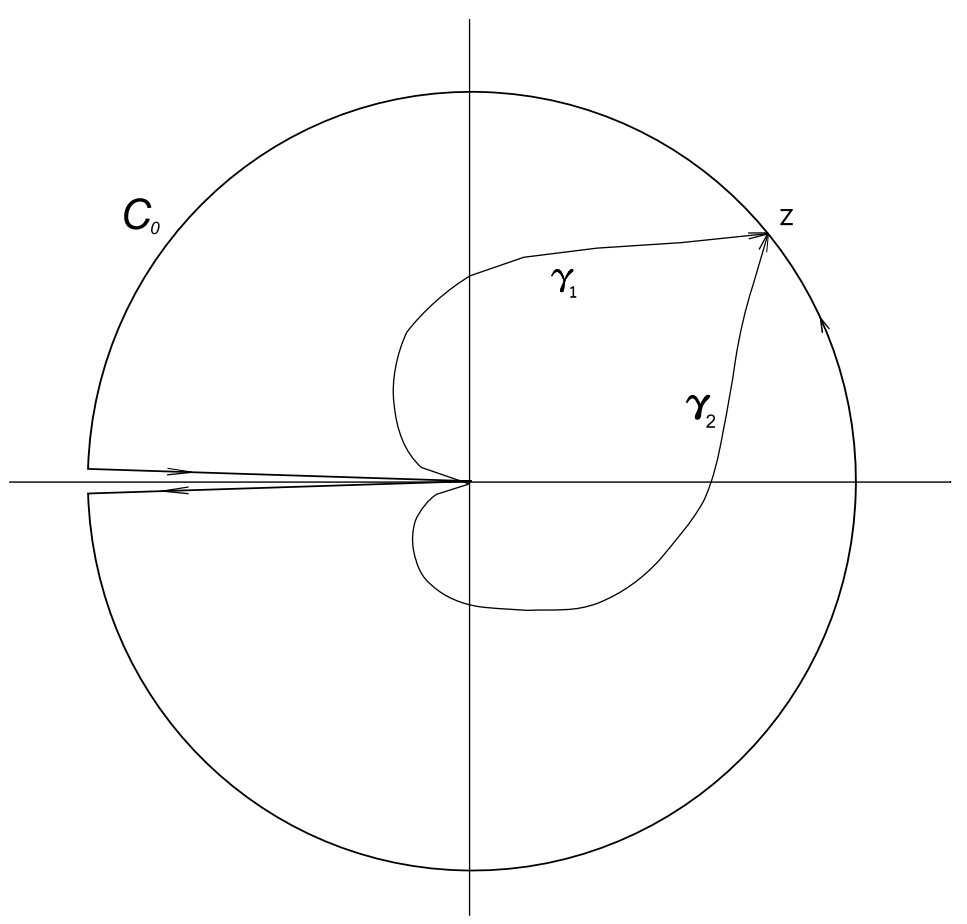

Figure 4: Integration paths in the $z^{\prime}$-plane for the first toy model: $\operatorname{arcs} \gamma_{1}$ and $\gamma_{2}$ going from $z_{0}=0$ to $z$ relate to (50); loop $C_{0}$ is the integration path in (56).

is analytical in the whole complex plane $z$ for such $\epsilon(z)$. Indeed, the left-hand side of (45) is analytical inside the circle $|z|=1$, since $g_{+}(z)$ is analytical there, and $\epsilon(z)$ is also analytical at $|z|<1$ except from the simple pole in the origin $z=0$, where $g_{+}(z)$ takes zero value. Similarly, the right-hand side of (45) is analytical outside the circle $|z|=1$. Therefore $U(z)$ is a constant, and we can put $U(z) \equiv 1$. The function $g_{+}(z)$ is determined by (50), with

$$
z_{0}=0, \quad U(z) \equiv 1, \quad i \mathcal{F}(z)=-\lambda \log z-\frac{1}{2}\left(z-z^{-1}\right) .
$$

We have set the initial integration point $z_{0}$ to zero in (50) to provide $g_{+}(0)=$ 0 . Further, integration path in $z^{\prime}$-plane should approach the point $z_{0}=0$ from the left half-plane $\operatorname{Im} z^{\prime}<0$ to guarantee convergence of the integral in $z^{\prime}$ in the origin. Two integration paths $\gamma_{1}$ and $\gamma_{2}$, which satisfy all above 


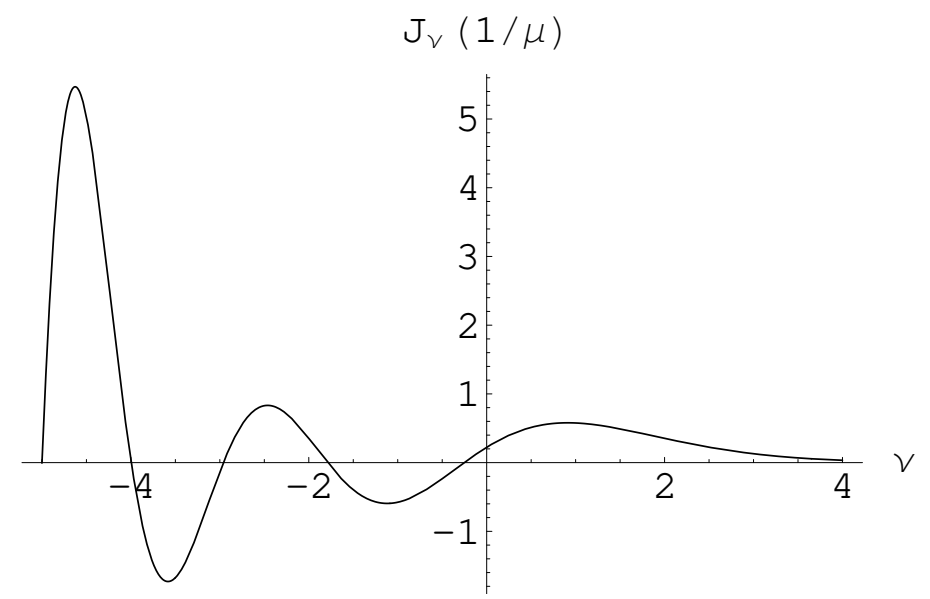

Figure 5: Plot of Bessel function $J_{\nu}(1 / \mu)$ versus $\nu$ at $\mu=0.5$.

conditions, are shown in Fig. 4. Integration in (50) along each path, either $\gamma_{1}$ or $\gamma_{2}$, should define the same function $g_{+}(z)$. This requirement will be satisfied, if

$$
I(\lambda)=0,
$$

where

$$
I(\lambda)=\int_{C_{0}} \frac{d z^{\prime}}{z^{\prime}} \exp \left\{\frac{i}{\mu} \mathcal{F}\left(z^{\prime}\right)\right\},
$$

and the integration path $C_{0}=\gamma_{2}-\gamma_{1}$ is shown in Fig. 4. Condition (56) determines the spectrum $\lambda$ for our first Toy model. After change of the integration variable $z^{\prime}=u^{-1}$, the right-hand side in (57) reduces (up to the sign) to the integral, which gives the well-known representation of the Bessel function,

$$
\int_{-\infty}^{\left(0^{+}\right)} \frac{d u}{u^{\nu+1}} \exp \left[a\left(u-u^{-1}\right) / 2\right]=2 \pi i J_{\nu}(a)
$$

with $\nu=-\lambda / \mu, a=1 / \mu$. Integration contour in (58) "starts at infinity on the negative real $u$-axis, encircles the origin counter-clockwise, and returns to its starting point", see formula (5) in page 15 in [2]. So, the eigenvalues $\lambda_{n}$ 

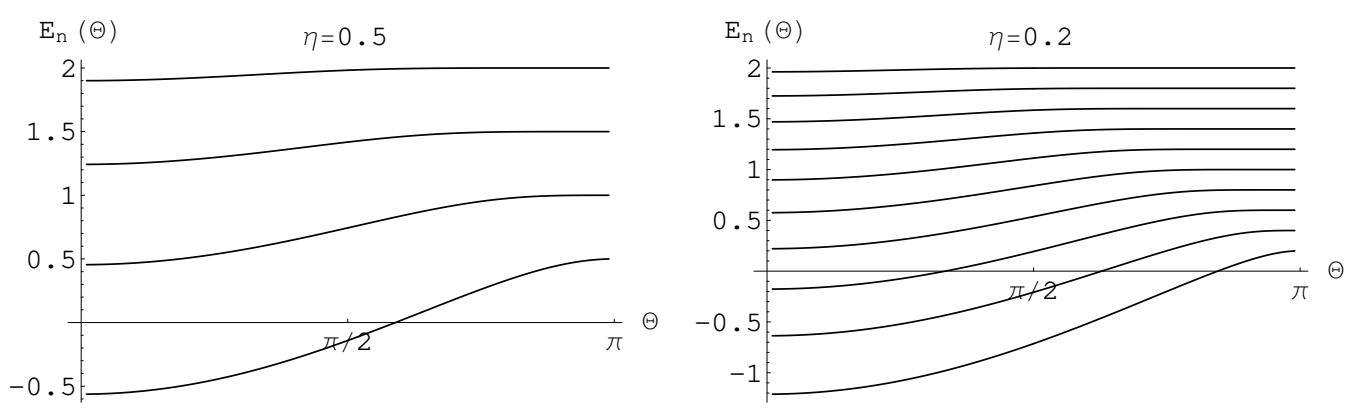

Figure 6: Dispersion curves $E_{n}(\Theta)$ for the first toy model for $\chi=0.5$ (left) and $\chi=0.2$ (right) according to (60).

of equation (43) with $\epsilon(z)=\epsilon_{1}(z)$ are determined by zeros $\nu_{n}$ of the Bessel function $J_{\nu}(1 / \mu)$ as the function of its order $\nu$ :

$$
\lambda_{n}=-\nu_{n} \mu, \quad J_{\nu_{n}}(1 / \mu)=0 .
$$

A plot of $J_{\nu}(1 / \mu)$ versus $\nu$ at $\mu=0.5$ is shown in Fig. 5. For the energy spectrum $E_{n}(\Theta)$ in the first Toy model equations (59), (54) yield

$$
\begin{aligned}
& E_{n}(\Theta)=-\nu_{n} \chi \\
& \text { where } J_{\nu_{n}}\left[\frac{2 \cos (\Theta / 2)}{\chi}\right]=0, \quad n=1,2, \ldots
\end{aligned}
$$

Fig. 6] shows the lowest dispersion curves $E_{n}(\Theta)$ for $\chi=0.5$ and $\chi=0.2$. Note, that $E_{n}(\pi)=n \chi$ in this model.

It is straightforward to extract the small- $\mu$ asymptotics from above exact results. In the $\mu \rightarrow+0$ limit the integral in (56) is determined by the saddle points $z_{ \pm}=-\lambda \pm i \sqrt{1-\lambda^{2}}$ of the function $\mathcal{F}\left(z^{\prime}\right)$. They lie on the circle $\left|z^{\prime}\right|=1$, if $|\lambda|<1$.

If $|\lambda|$ is far enough from 1 , they provide two separate complex conjugate contributions to the integral in (57). In the leading order in $\mu$, this yields

$$
\lambda_{n} \theta_{a}+\sin \theta_{a}=\pi \mu\left(n-\frac{1}{4}\right), \quad \cos \theta_{a}=-\lambda_{n}
$$

in agreement with semiclassical quantization formula (27). 
If $\lambda$ approaches -1 , two saddle points $z_{ \pm}$merge at 1 , and the integral (57) becomes proportional to the Airy function [1]

$$
I(\lambda) \approx \int_{-\infty}^{\infty} d \theta \exp \left\{\frac{i}{\mu}\left[-(\lambda+1) \theta+\frac{\theta^{3}}{6}\right]\right\}=2 \pi(2 \mu)^{1 / 3} \operatorname{Ai}\left[-(\lambda+1)(2)^{1 / 3} \mu^{-2 / 3}\right]
$$

in the limit $\mu \rightarrow 0$. From (56) we get in this case

$$
\lambda_{n}=-1+\mu^{2 / 3} 2^{-1 / 3} z_{n}, \text { where } \operatorname{Ai}\left[-z_{n}\right]=0, \quad n=1,2, \ldots,
$$

in agreement with (31).

If $\lambda$ gets close to $+1, z_{ \pm}$approach -1 . However, there are still two saddle point contributions to $I(E)$, coming from the the upper and lower edges of the contour $C_{0}$ at $z^{\prime}=-1$, see Fig. 4. The resulting equation for $\lambda$ reads

$$
\tan \left(\frac{\pi \lambda}{\mu}\right)=-\frac{\operatorname{Ai}\left[(\lambda-1)\left(2 / \mu^{2}\right)^{1 / 3}\right]}{\operatorname{Bi}\left[(\lambda-1)\left(2 / \mu^{2}\right)^{1 / 3}\right]}
$$

where $\operatorname{Bi}(x)$ is the second Airy functions, which solves (together with $\operatorname{Ai}(x)$ )

the differential equation equation $y^{\prime \prime}(x)-x y(x)=0$, and has the following integral representation [1]

$$
(3 a)^{-1 / 3} \pi \operatorname{Bi}\left[ \pm(3 a)^{-1 / 3} x\right]=\int_{0}^{\infty} d t\left[\exp \left(-a t^{3} \pm x t\right)+\sin \left(a t^{3} \pm x t\right)\right] .
$$

Equation (63) describes the crossover at $\lambda \approx 1$ of the $\mu \rightarrow 0$ asymptotics from the semiclassical regime (61) at $\lambda<1$, to $\lambda_{n} \approx \mu n$ at $\lambda>1$.

\subsection{Toy model 2}

In the first Toy model with $\omega(\theta)=-\cos \theta$, variation of the total momentum $\Theta$ only rescales $\epsilon(\theta ; \Theta)$, see $(\underline{52})$. However, for the real free-fermion dispersion law (9), the topology of $\epsilon(\theta ; \Theta)$ also changes with increasing $\Theta$, compare Figs. 2 and 3. To mimic this property, let us modify the "free-fermion dispersion law" to

$$
\omega_{2}(\theta)=-\cos \theta-\gamma \cos (2 \theta) .
$$

with positive $\gamma$. Then the function (17) takes the form

$$
\epsilon(\theta ; \Theta)=-2 \cos (\Theta / 2) \cos \theta-2 \gamma \cos (\Theta) \cos (2 \theta)
$$


Equation (41) reads now

$$
\begin{aligned}
& \left.\left\{\left[-\left(z+z^{-1}\right) \cos (\Theta / 2)-\left(z^{2}+z^{-2}\right) \gamma \cos \Theta\right]-E(\Theta)\right]\right\} \phi(z) \\
& =-\chi z f_{S_{1}} \frac{d z^{\prime}}{\pi i} \frac{\phi\left(z^{\prime}\right)}{\left(z^{\prime}-z\right)^{2}} .
\end{aligned}
$$

After rescaling

$$
\lambda=\frac{E(\Theta)}{\cos (\Theta / 2)}, \quad \mu=\frac{\chi}{\cos (\Theta / 2)}, \quad \rho=\frac{\gamma \cos \Theta}{\cos (\Theta / 2)},
$$

it reduces to (43) with $\epsilon(z)=\epsilon_{2}(z)=-\left(z+z^{-1}\right)-\rho\left(z^{2}+z^{-2}\right)$.

One can naturally come to this Toy model approximating the exact freefermion dispersion law (9) by three initial terms of its Fourier expansion. This approximation is rather good, if $h_{x}$ is not too close to the quantum phase transition point $h_{x}=1$.

It follows from (45), that $U(z)$ can be now written as

$$
U(z)=A_{1}+A_{2}\left(z+z^{-1}\right),
$$

with two constants $A_{1}, A_{2}$. The function $g_{+}(z)$ determined by (50) takes the form

$$
g_{+}(z)=\int_{0}^{z} \frac{d z^{\prime}}{\mu z^{\prime}}\left[A_{1}+A_{2}\left(z^{\prime}+\frac{1}{z^{\prime}}\right)\right] \exp \left\{\frac{i}{\mu}\left[\mathcal{F}\left(z^{\prime}\right)-\mathcal{F}(z)\right]\right\},
$$

where

$$
i \mathcal{F}(z)=-\lambda \log z-\left(z-\frac{1}{z}\right)-\frac{\rho}{2}\left(z^{2}-\frac{1}{z^{2}}\right)
$$

To provide convergence of the integral in (69), the variable $z^{\prime}$ should approach the origin inside one of two allowed sectors, which are defined by the condition $\operatorname{Re}\left(\rho / z^{\prime 2}\right)<0$, or explicitly

$$
\begin{aligned}
& \pi / 4<\arg z^{\prime}<3 \pi / 4, \text { and }-3 \pi / 4<\arg z^{\prime}<-\pi / 4, \quad \text { if } \rho>0, \\
& -\pi / 4<\arg z^{\prime}<\pi / 4, \quad \text { and } \quad 5 \pi / 4<\arg z^{\prime}<3 \pi / 4, \quad \text { if } \rho<0 .
\end{aligned}
$$

Definition (69) becomes unambiguous and independent on the integration path between the points 0 and $z$, if the constants $A_{1}$ and $A_{2}$ solve two linear uniform equations:

$$
\begin{aligned}
& W_{11}(\lambda) A_{1}+W_{12}(\lambda) A_{2}=0 \\
& W_{21}(\lambda) A_{1}+W_{22}(\lambda) A_{2}=0
\end{aligned}
$$




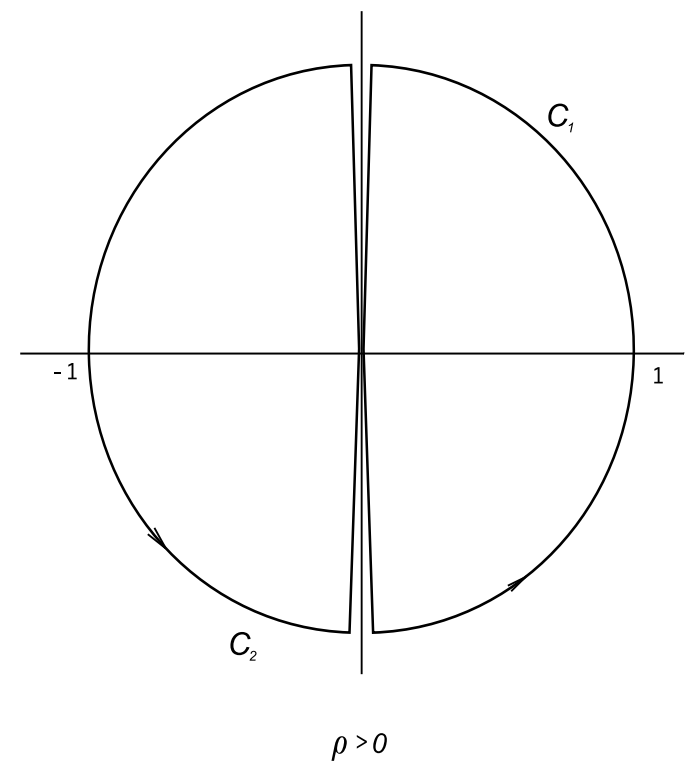

(a)

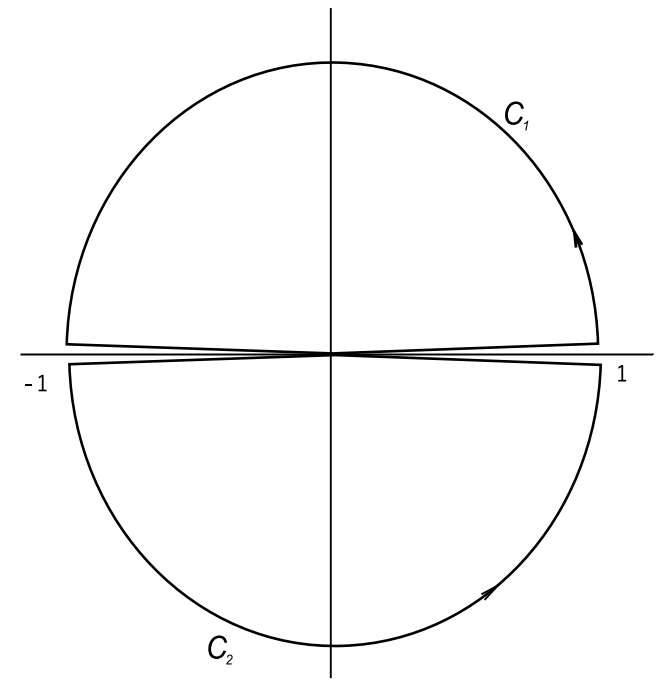

$\rho<0$

(b)

Figure 7: Integration contours $C_{1}$ and $C_{2}$ in (70): (a) for $\rho>0$, (b) $\rho<0$. Both $C_{1}$ and $C_{2}$ start and finish in the origin.

where

$$
\begin{aligned}
W_{i j}(\lambda) & =\int_{C_{i}} \frac{d z^{\prime}}{z^{\prime}} w_{j}\left(z^{\prime}\right) \exp \left[\frac{i}{\mu} \mathcal{F}\left(z^{\prime}\right)\right], \quad i, j=1,2 \\
w_{1}\left(z^{\prime}\right) & =1, \quad w_{2}\left(z^{\prime}\right)=z^{\prime}+\frac{1}{z^{\prime}}
\end{aligned}
$$

Integration contours $C_{1}$ and $C_{2}$ for the two cases $\rho>0$ and $\rho<0$ are shown in Fig. 7. We fix the branch of $\mathcal{F}\left(z^{\prime}\right)$ in (70) by the condition $\mathcal{F}(1)=0$.

Eigenvalues $\lambda_{n}$ in the second Toy model are then determined by the requirement:

$$
W_{11}\left(\lambda_{n}\right) W_{22}\left(\lambda_{n}\right)-W_{12}\left(\lambda_{n}\right) W_{21}\left(\lambda_{n}\right)=0
$$

With known $\lambda_{n}$, we obtain finally $E_{n}(\Theta)$ by use of (68).

Note. In the same way one can find exact solutions of equation (43) with $\epsilon(z)=\sum_{k=1}^{K} c_{k}\left(z^{k}+z^{-k}\right)$. In this case the eigenvalues $\lambda_{n}$ of (43) are determined from $\operatorname{det} W\left(\lambda_{n}\right)=0$, where $W(\lambda)$ denotes a $K \times K$-matrix. 
The small- $\mu$ behavior of the second Toy model is more rich than in the previous case. It is determined by the location of four saddle points of the integrals in (70). These saddle points are the solutions of the equation $\epsilon_{2}(z)=$ $\lambda$. We skip further discussions of the $\mu \rightarrow 0$ asymptotics of the Toy model 2 , since it is very similar to that of original equation (41), which will be described in the following Section.

\section{Weak coupling expansion}

In this section we return to the original equation (41), describing boundspinons in the two-fermion approximation, and obtain its perturbative solution in the weak coupling limit $\chi \rightarrow 0$. Several perturbative schemes have been developed for the analogous problem in IFT $[8,10,18]$. Here we shall use a different procedure, which utilizes results of Section 5 .

First, let us rewrite (41) in notations of Section 5 .

$$
[\epsilon(z)-\lambda] \phi(z)=-\mu z f_{S_{1}} \frac{d z^{\prime}}{\pi i} \frac{\phi\left(z^{\prime}\right)}{\left(z^{\prime}-z\right)^{2}},
$$

where

$$
\begin{aligned}
\epsilon(z) & =2 \sqrt{h_{x}}\left\{\left[h_{x}+\frac{1}{h_{x}}-z v-\frac{1}{z v}\right]^{1 / 2}+\left[h_{x}+\frac{1}{h_{x}}-\frac{z}{v}-\frac{v}{z}\right]^{1 / 2}\right\} \\
\mu & =\chi, \quad \lambda=E(\Theta), \quad v=\exp (i \Theta / 2)
\end{aligned}
$$

Remind, that $S_{1}$ is the unit circle, and $\phi(1 / z)=-\phi(z)$.

Function $\epsilon(z)$ has six branching points $0, h_{x} v, h_{x} v^{-1}, h_{x}^{-1} v, h_{x}^{-1} v^{-1}, \infty$. Its Riemann surface $\mathcal{L}$ has four sheets $\mathcal{L}_{\alpha, \beta}$ with $\alpha, \beta=+,-$, which are distinguished by the signs of the first an the second terms in braces in (72) at $z=1$. To separate the sheets, we draw four cuts in the $z$-plane shown in Fig. 8. Equation (71) is written in the sheet $\mathcal{L}_{+,+}$, which we shall call "the physical sheet".

Equations (44), (45) define functions $g_{ \pm}(z)$ and $U(z)$ in $\mathcal{L}_{+,+}$. It follows from these equations and (72), that all singularities of $U(z)$ in the physical sheet are the square root singularities of $\epsilon(z)$. Therefore, $U(z)$ is finite in $\mathcal{L}_{+,+}$.

Let us turn now to the integral formula (50). As in the previous Section, we should put $z_{0}=0$ in it to provide $g_{+}(0)=0$. The function $\mathcal{F}(z)$, determined by (51), is singular in the physical sheet at $z \rightarrow 0, \mathcal{F}(z) \sim z^{-1 / 2}$. 


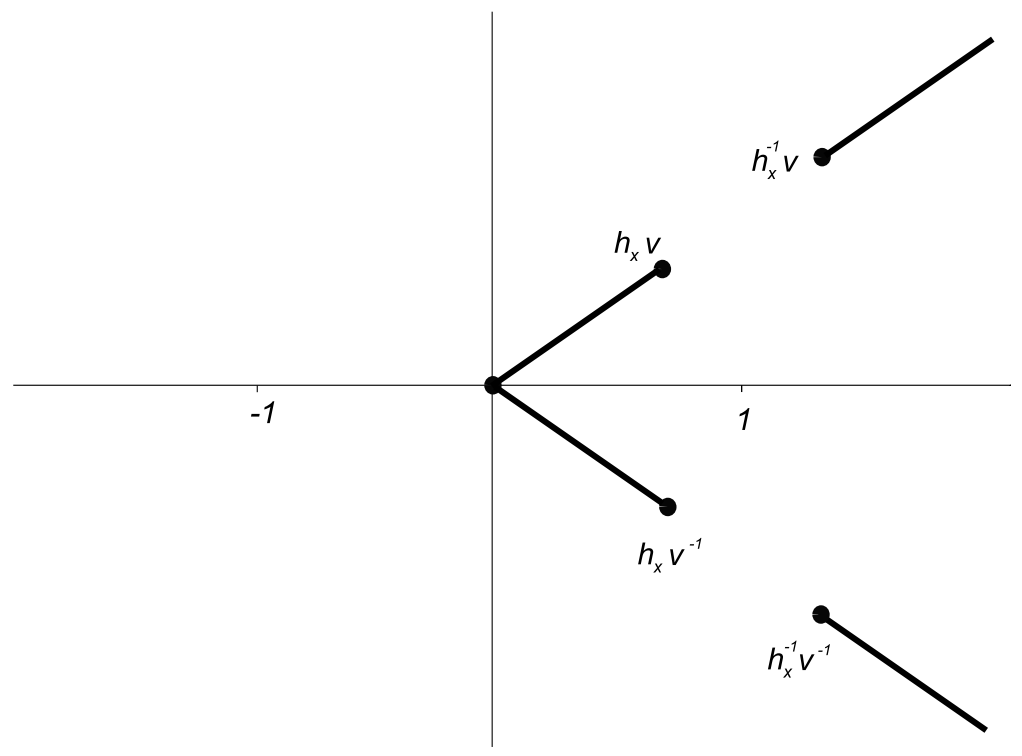

Figure 8: Square root branching points of function $\epsilon(z)$ determined by (72), and cuts on $z$-plane.

Nevertheless, the integral in (50) converges, if the whole integration path lies in $\mathcal{L}_{+,+}$, approaching to the origin either from the right, or from the left side. This follows from the fact, that $\epsilon(z)$ is positive at real $z$ in the physical sheet.

Furthermore, three integral equalities must be satisfied

$$
\int_{C_{\alpha}} \frac{d z}{z} U(z) \exp \left\{\frac{i}{\mu} \mathcal{F}(z)\right\}=0, \quad \alpha=0,1,2,
$$

where the integration path $C_{0}$ is shown in Fig. 4, and paths $C_{1,2}$ are drawn in Fig. 7(b). Note, that only two conditions in (74) are independent, since $C_{0}=C_{1}+C_{2}$. Conditions (74) are necessary to guarantee, that: 
- the integral in (50) defines a single valued function in the unit circle $S_{1}$

- $g_{+}(z)$ given by (50) vanishes, when $z$ approaches to the origin in $\mathcal{L}_{+,+}$ either from the left, or from the right side of the cuts, see Fig. 8 .

In the limit $\mu \rightarrow+0$ the integrals in (174) are determined by contributions of the saddle point of function $\mathcal{F}(z)$, i.e. by the solutions of equation

$$
\epsilon(z)=\lambda .
$$

This equation has four solutions $z_{a}, z_{b}, z_{a}^{-1}, z_{b}^{-1}$ in the Riemann surface $\mathcal{L}$, which are given by

$$
\begin{aligned}
z_{\alpha}+z_{\alpha}^{-1} & =\frac{\lambda^{2} \cos (\Theta / 2) \pm\left\{\left[\lambda^{2}-16 \sin ^{2}(\Theta / 2)\right]\left[\lambda^{2}-16 h_{x}^{2} \sin ^{2}(\Theta / 2)\right]\right\}^{1 / 2}}{8 h_{x} \sin ^{2}(\Theta / 2)} \\
\alpha & =a, b .
\end{aligned}
$$

Important for us are the saddle points, which lie on the circle $|z|=1$, or are close to it. In the simplest case, there are only two such solutions of (75): $z_{a}=\exp \left(i \theta_{a}\right)$, and $z_{a}^{-1}=\exp \left(-i \theta_{a}\right)$. Suppose, they are well separated, being far enough from 1 and -1 . Then the saddle point expansion of the integral in (74) over the path $C_{0}$ leads to the asymptotic expansion

$$
-\mathcal{F}\left(\theta_{a}\right)=\mu \pi\left(n-\frac{1}{4}\right)+\mu \arg \left\langle U\left(\theta_{a}+\delta \theta\right) \exp \left[\frac{i}{\mu} \Delta \mathcal{F}\left(\theta_{a}+\delta \theta\right)\right]\right\rangle,
$$

valid to all orders in $\mu$. Here we use short notations $\mathcal{F}(\theta)=\mathcal{F}[z(\theta)], U(\theta)=$ $U[z(\theta)]$, and

$$
\Delta \mathcal{F}\left(\theta_{a}+\delta \theta\right)=\mathcal{F}\left(\theta_{a}+\delta \theta\right)-\mathcal{F}\left(\theta_{a}\right)-\frac{\mathcal{F}^{\prime \prime}\left(\theta_{a}\right)}{2} \delta \theta^{2} .
$$

Averaging $\langle f(\delta \theta)\rangle$ implies formal term-by term integration

$$
\langle f(\delta \theta)\rangle=\frac{\int_{-\infty}^{\infty} d \delta \theta f(\delta \theta) \exp \left[\frac{i \mathcal{F}^{\prime \prime}\left(\theta_{a}\right)}{2 \mu} \delta \theta^{2}\right]}{\int_{-\infty}^{\infty} d \delta \theta \exp \left[\frac{i \mathcal{F}^{\prime \prime}\left(\theta_{a}\right)}{2 \mu} \delta \theta^{2}\right]},
$$


with $f(\delta \theta)$ expanded into a power series in $\delta \theta$. Under appropriate normalization of $\phi(z)$, the function $U\left(\theta_{a}+\delta \theta\right)$ can be expanded as

$$
U\left(\theta_{a}+\delta \theta\right)=1+\sum_{i=1}^{\infty} \sum_{l=0}^{\infty} c_{i l} \mu^{i} \delta \theta^{l} .
$$

To obtain the coefficients $c_{i l}$ explicitly, one should write down the Neumann series, giving the formal solution of equation (171) in the class of generalized functions,

$$
\begin{aligned}
\phi(\theta) & =\phi^{(0)}(\theta)+\phi^{(1)}(\theta)+O\left(\mu^{2}\right) \\
\phi^{(0)}(\theta) & =C\left[\delta\left(\theta-\theta_{a}\right)-\delta\left(\theta+\theta_{a}\right)\right] \\
\phi^{(1)}(\theta) & =\frac{\mu C}{4 \pi[\epsilon(\theta)-\lambda]}\left\{\frac{1}{\sin ^{2}\left[\left(\theta-\theta_{a}\right) / 2\right]}-\frac{1}{\sin ^{2}\left[\left(\theta+\theta_{a}\right) / 2\right]}\right\} .
\end{aligned}
$$

Substitution of this expansion into (47) gives us a smooth function $U(\theta)$ as a power series in $\mu$

$$
\begin{aligned}
U(\theta) & =U^{(0)}(\theta)+U^{(1)}(\theta)+O\left(\mu^{2}\right), \\
U^{(0)}(\theta) & =\frac{C}{2 \pi}\left[\epsilon(\theta)-\epsilon\left(\theta_{a}\right)\right]\left[\frac{1}{1-\exp \left[i\left(\theta-\theta_{a}\right)\right]}-\frac{1}{1-\exp \left[i\left(\theta+\theta_{a}\right)\right]}\right],
\end{aligned}
$$

which in turn can be expanded in $\theta$ at $\theta=\theta_{a}$. The result is brought to the form (177), if one puts $C=-2 \pi i / \epsilon^{\prime}\left(\theta_{a}\right)$.

The perturbation procedure described above should be modified, if equation (75) has four solutions $z_{a}, z_{b}, z_{a}^{-1}, z_{b}^{-1}$ on the circle $|z|=1, z_{a, b}=$ $\exp \left[i \theta_{a, b}\right]$, see Fig. 3. If they are well separated, the zero-order function $\phi^{(0)}(\theta)$ in $(78)$ can be written as

$$
\phi^{(0)}(\theta)=C_{a}\left[\delta\left(\theta-\theta_{a}\right)-\delta\left(\theta+\theta_{a}\right)\right]+C_{b}\left[\delta\left(\theta-\theta_{b}\right)-\delta\left(\theta+\theta_{b}\right)\right] .
$$

Using the leading saddle point approximation for the integrals in (74) with $j=1,2$, we obtain a system of two equations

$$
\begin{aligned}
& u_{a}\left[\frac{2 \pi \mu}{\epsilon^{\prime}\left(\theta_{a}\right)}\right]^{1 / 2} e^{i \pi / 4+i \mathcal{F}\left(\theta_{a}\right) / \mu}+u_{b}\left[\frac{2 \pi \mu}{-\epsilon^{\prime}\left(\theta_{b}\right)}\right]^{1 / 2} e^{-i \pi / 4+i \mathcal{F}\left(\theta_{b}\right) / \mu}=0, \\
& u_{a}\left[\frac{2 \pi \mu}{\epsilon^{\prime}\left(\theta_{a}\right)}\right]^{1 / 2} e^{-i \pi / 4-i \mathcal{F}\left(\theta_{a}\right) / \mu}+u_{b}\left[\frac{2 \pi \mu}{-\epsilon^{\prime}\left(\theta_{b}\right)}\right]^{1 / 2} e^{i \pi / 4-i \mathcal{F}\left(\theta_{b}\right) / \mu}=0,
\end{aligned}
$$


for constants $u_{a}=U^{(0)}\left(\theta_{a}\right), u_{b}=U^{(0)}\left(\theta_{b}\right)$, which are simply related with $C_{a}, C_{b}$. Setting its determinant to zero, we get

$$
\mathcal{F}\left(\theta_{b}\right)-\mathcal{F}\left(\theta_{a}\right)=\pi \mu\left(n-\frac{1}{2}\right)+O\left(\mu^{2}\right),
$$

in agreement with the semiclassical formula (29).

It is easy to determine the eigenvalues $\lambda_{n}$ of (71) in the limit $\mu \rightarrow+0$ for $\lambda>\epsilon(\theta=\pi)$. Let us rewrite condition (74) with $\alpha=0$ as

$$
\begin{aligned}
& 0=\int_{-\pi}^{\pi} d \theta U(\theta) \exp [i \mathcal{F}(\theta) / \mu]+\int_{\pi}^{\pi+i \infty} d \theta U(\theta) \exp [i \mathcal{F}(\theta) / \mu]+ \\
& \int_{-\pi-i \infty}^{-\pi} d \theta U(\theta) \exp [i \mathcal{F}(\theta) / \mu]=\int_{-\pi}^{\pi} d \theta U(\theta) \exp [i \mathcal{F}(\theta) / \mu]+ \\
& \{1-\exp [-i \mathcal{F}(2 \pi) / \mu]\} \int_{\pi}^{\pi+i \infty} d \theta U(\theta) \exp [i \mathcal{F}(\theta) / \mu]
\end{aligned}
$$

In the second equality we have taken into account, that $U(\theta-2 \pi)=U(\theta)$, and $\mathcal{F}(\theta-2 \pi)=\mathcal{F}(\theta)-\mathcal{F}(2 \pi)$.

If $\lambda>\epsilon(\theta=\pi)$, the single saddle point $z_{a}$ in the integration path $C_{0}$ is located in the interval $(-1,0)$ on the real $z$-axis. Two contributions of this saddle point will cancel each other in (83), if $\mathcal{F}(2 \pi)=-2 \pi n \mu$ with integer $n$, i.e.

$$
\lambda_{n}=\frac{1}{2 \pi} \int_{0}^{2 \pi} d \theta \epsilon(\theta)+\mu n .
$$

This asymptotic formula is valid to all orders 3 in $\mu$. Rewriting it in original variables (73) and using (17), one obtains the bound-spinon spectrum

$$
\begin{aligned}
& E_{n}(\Theta)=\frac{1}{2 \pi} \int_{0}^{2 \pi} d \theta 2 \omega(\theta)+\chi n \\
& \text { for } E_{n}(\Theta)>\epsilon(\pi, \Theta) \text { and } \chi \rightarrow+0 .
\end{aligned}
$$

This result has a clear physical interpretation. Condition (86) means, that a bound-spinon can be considered semiclassically as a large enough domain, bounded by two domain walls. These domain walls move back and forth

\footnotetext{
${ }^{3}$ Corrections to (84) of order $\mu \exp [-A(\lambda) / \mu]$ can be obtained from (88).
} 
without collisions according to the classical equations of motion corresponding to the Hamiltonian (14). The two terms on the right-hand side of (85) give the kinetic and potential energy of domain walls averaged over their oscillations. So, the energy of such a bound-spinon does not depend on its momentum $\Theta$, and hence, it can not move as a whole along the spin chain.

The asymptotic formulas (76), (82), (84) describe the small- $\mu$ behavior of eigenvalues $\lambda_{n}$ of (71) for generic values of $\lambda$ and $\Theta$. Five crossover regimes are realized, when $\lambda$ approaches the critical values of $\epsilon(\theta)$ and two or four solutions of equation (75) merge. All these regimes can be analyzed by means of almost the same perturbation procedure utilizing equations (47) and (74). Minor modifications in it are caused by further degeneracy of $\mathcal{F}(\theta)$ near the saddle point $\theta_{0}: \mathcal{F}(\theta)-\mathcal{F}\left(\theta_{0}\right) \sim\left(\theta-\theta_{0}\right)^{j}$ where $j=3$ or $j=5$ instead of $j=2$ in the case of Morse saddle point considered previously. In the $\mu \rightarrow 0$ limit this leads to perturbation expansions for $\lambda_{n}$ in powers of parameter $\mu^{1 / 3}$ or $\mu^{1 / 5}$. Below we present the leading terms of these expansions, skipping the details of calculations.

1. $\theta_{a} \approx 0,0<\Theta<\Theta_{m}$, where $\Theta_{m}=2 \arccos h_{x}$,

$$
\lambda_{n}=\epsilon(0)+z_{n} \mu^{2 / 3}\left[\epsilon^{\prime \prime}(0) / 2\right]^{1 / 3} .
$$

2. $\theta_{a} \approx \pi, 0<\Theta<\pi$,

$$
\tan \left[\frac{\mathcal{F}(\pi)}{\mu}\right]=\frac{\operatorname{Ai}\left[\left[\lambda_{n}-\epsilon(\pi)\right]\left[-2 / \epsilon^{\prime \prime}(\pi)\right]^{1 / 3} \mu^{-2 / 3}\right]}{\operatorname{Bi}\left[\left[\lambda_{n}-\epsilon(\pi)\right]\left[-2 / \epsilon^{\prime \prime}(\pi)\right]^{1 / 3} \mu^{-2 / 3}\right]}
$$

3. $\Theta_{m}<\Theta<\pi$

(a) $\theta_{a} \approx \theta_{b} \approx \theta_{0}>0$, where $\cos \theta_{0}=\frac{\cos (\Theta / 2)}{\cos \left(\Theta_{m} / 2\right)}$.

$$
\begin{aligned}
& \lambda_{n, 1}=\epsilon\left(\theta_{0}\right)+z_{n} \mu^{2 / 3}\left[\epsilon^{\prime \prime}\left(\theta_{0}\right) / 2\right]^{1 / 3}, \\
& \lambda_{n, 2}=\epsilon\left(\theta_{0}\right)+z_{n}^{\prime} \mu^{2 / 3}\left[\epsilon^{\prime \prime}\left(\theta_{0}\right) / 2\right]^{1 / 3},
\end{aligned}
$$

where $n=1,2, \ldots$

(b) $\theta_{b} \approx 0, \theta_{a}>0$,

$$
\operatorname{cotan}\left[-\frac{\mathcal{F}\left(\theta_{a}\right)}{\mu}-\frac{\pi}{4}\right]=\frac{\operatorname{Ai}\left[\left[\lambda_{n}-\epsilon(0)\right]\left[-2 /\left|\epsilon^{\prime \prime}(0)\right|\right]^{1 / 3} \mu^{-2 / 3}\right]}{\operatorname{Bi}\left[\left[\lambda_{n}-\epsilon(0)\right]\left[-2 /\left|\epsilon^{\prime \prime}(0)\right|\right]^{1 / 3} \mu^{-2 / 3}\right]} .
$$


4. $\Theta=\Theta_{m}, \theta_{a} \approx 0, \theta_{b} \approx i \theta_{a}$,

$$
\lambda_{n}=\left.\left\{\epsilon(\theta)+\mu^{4 / 5}\left[\frac{\partial^{4} \epsilon(\theta) / \partial \theta^{4}}{6}\right]^{1 / 5} c_{n}\right\}\right|_{\theta=0},
$$

where $\epsilon(0)=2\left(1-h_{x}^{2}\right)^{1 / 2}, \partial^{4} \epsilon(\theta) /\left.\partial \theta^{4}\right|_{\theta=0}=h_{x}^{2} / \sqrt{1-h_{x}^{2}}$, and $n=$ $1,2, \ldots$

The numbers $z_{n}$ and $z_{n}^{\prime}$ are consecutive zeroes of $\mathrm{Ai}(-z)$ and $\mathrm{Ai}^{\prime}(-z)$ respectively, and $c_{n}$ are the solutions of equation

$$
\begin{aligned}
& \int_{0}^{\infty} d y\left[\sin \left(\frac{y^{5}}{20}-y c_{n}\right)-\exp \left(-\frac{y^{5}}{20}+y c_{n}\right)\right] . \\
& \int_{0}^{\infty} d x x^{2} \cos \left(\frac{x^{5}}{20}-x c_{n}\right)=\int_{0}^{\infty} d x \cos \left(\frac{x^{5}}{20}-x c_{n}\right) . \\
& \int_{0}^{\infty} d y y^{2}\left[\sin \left(\frac{y^{5}}{20}-y c_{n}\right)+\exp \left(-\frac{y^{5}}{20}+y c_{n}\right)\right]
\end{aligned}
$$

$c_{1}=1.787, c_{2}=3.544, c_{3}=5.086$.

Note, that all asymptotic regimes described above are realized also in the second Toy model, and the four regimes described by equations (76), (84), (87), (88) are relevant to the first Toy model as well.

\section{Discussion}

In this paper we apply a technique developed in IFT to study the effect the discreteness of the lattice on the kink confinement in a non-critical onedimensional system with explicitly broken $\mathbb{Z}_{2}$-symmetry. We study the model of the Ising spin-1/2 chain ferromagnet at zero temperature in the presence of a skew magnetic field, as a particular realization of such a system. The magnetic field $h_{x}$, transverse to the easy $z$-axis, serves to induce the quantum phase transition at $h_{x}= \pm 1$ and to allow kinks in the ordered phase $\left|h_{x}\right|<$ 1 to move. The longitudinal field $h_{z}$ breaks the $\mathbb{Z}_{2}$-symmetry and leads to confinement of kinks into pairs, the bound-spinons. We calculate the dispersion law $E_{n}(\Theta)$ of bound-spinons in the limit of small $h_{z}$ in the leading order in this parameter.

The dispersion law of bound-spinons can be understood within a simple heuristic picture, in which one treats a bound-spinon as bound state of 
two classical particles (kinks) attracting each other with a linear potential proportional to $h_{z}$. If these particles are close enough to each other, such that they can meat during their classical motion, then they can drift as a whole along the spin chain. In this case, one can determine the dispersion law $E_{n}(\Theta)$ of a bound-spinon from the Bohr-Sommerfeld quantization rule, if $n \gg 1$.

On the other hand, large enough bound-spinons can be viewed as two independent well separated kinks. The linear potential leads to localization of an isolated kink in the discrete spin chain, similarly to localization of an electron moving in a periodic potential by a uniform electric field [24]. The classical motion of isolated kinks looks like oscillations around certain positions in the chain. If two kinks forming a bound-spinon do not meet during their classical oscillations, the velocity $v_{n}(\Theta)$ of the bound-spinon is zero. Since $v_{n}(\Theta)=\partial E_{n}(\Theta) / \partial \Theta$, we conclude $E_{n}(\Theta)$ is constant for such a bound-spinon. It is clear that the energy spectrum should be equidistant in this region

$$
E_{n+1}(\Theta)-E_{n}(\Theta)=2 h_{z} \bar{\sigma},
$$

since the bound-spinon state $\left|\Phi_{n+1}(\Theta)\right\rangle$ contains one extra inverted spin in the domain bounded by two kinks, compared to the state $\left|\Phi_{n}(\Theta)\right\rangle$.

A more systematic theory is based on the perturbative analysis of the singular integral equation (41), which is analogous to the Bethe-Salpeter equation in IFT . The equations have been obtained in the two-fermion approximation and become exact in the limit $h_{z} \rightarrow 0$. The bound-spinon energy spectrum $E_{n}(\Theta)$ is determined in this approximation as eigenvalues of equation (41). We develop perturbation theory for this equation in small $h_{z}$ and, concentrating on its leading order, describe eight asymptotic regimes for the bound-spinon energy spectra $E_{n}(\Theta)$, instead of two regimes (3) and (4) known in IFT.

It was shown [8], that the IFT Bethe-Salpeter equation reproduces the energy spectra of stable "mesons" with reasonable accuracy not only in the limit $h \rightarrow 0$, but also at finite, and even at large values of the magnetic field $h$. If this situations holds in the discrete-lattice case, it would be reasonable to study the spectrum of equation (41) at finite $h_{z} \sim 1$ as well. Few steps in this direction give exact solutions of its simplified versions (53) and (67), which were described in Section 6 .

In conclusion let us comment on the higher order corrections to the obtained bound-spinon spectrum $E_{n}(\Theta)$ in the limit $h_{z} \rightarrow 0$. It is straightfor- 
ward to determine the higher order terms in the weak coupling expansion of the eigenvalues of equation (41), as it was described in Section 7 . However, one should also take into account corrections to equation (41), which is an approximation by itself. First, the kernel $G_{\Theta}\left(\theta, \theta^{\prime}\right)$ in the integral in equation (37) has a regular part $G_{\Theta}^{(r e g)}\left(\theta, \theta^{\prime}\right)$ omitted in (41). Only few modifications in the perturbation schema of Section 7 are needed to apply it to equation (37) with this term restored. Second, starting from the second order in $h_{z}$ one should take into multi-fermion corrections, which arise from the four-fermion, six-fermion, ..., contributions to the bound-spinon eigenvector $\left|\Phi_{n}(\Theta)\right\rangle$. As we know from IFT $[8,10,18]$, multi-fermion effects lead to renormalization of the fermion dispersion law $\omega(\theta)$ and coupling constant $2 h_{z} \bar{\sigma}$, and should lead to the decay of unstable bound-spinons. All these effect are lost in the twofermion approximation (34), but contribute to the bound-spinon dispersion law $E_{n}(\Theta)$ in higher orders in $h_{z}$. A perturbative analysis of multi-fermion effects in the discrete Ising model (7) is an interesting problem, which remains for future work.

\section{Acknowledgements}

I am thankful to the Institute of Theoretical Physics of the University of Münster for hospitality. I would like to express my gratitude to Gernot Münster for many helpful discussions and valuable remarks. This work is supported by Deutsche Forschungsgemeinschaft (DFG) under the grant

$\mathrm{Mu} 757 / 15-1$ and by the Belarusian Republican Foundation for Fundamental Research under the grant $\Phi 07-147$.

\section{References}

[1] Abramowitz, M., Stegun, I.A.: Handbook of Mathematical Functions. Dover (1965)

[2] Bateman, H.: Higher Transcendental Functions, Volume II. McGrawHill, New York (1953)

[3] Bhaseen, M.J., Tsvelik, A.M.: Aspects of confinement in low dimensions. cond-mat/0409602 (2004) 
[4] Delfino, G.: Integrable field theory and critical phenomena: the Ising model in a magnetic field. J. Phys. A 37(14), R45-R78 (2004)

[5] Delfino, G., Grinza, P., Mussardo, G.: Decay of particles above threshold in the Ising field theory with magnetic field. Nucl. Phys. B 737(3), 291$303(2006)$

[6] Delfino, G., Mussardo, G., Simonetti, P.: Non-integrable quantum field theories as perturbations of certain integrable models. Nucl. Phys. B 473(3), 469-508 (1996)

[7] Fogedby, H.C.: The Ising chain in a skew magnetic field. J. Phys. C 11(3-6), 2801-2813 (1978)

[8] Fonseca, P., Zamolodchikov, A.: Ising spectroscopy I: mesons at $T<T_{c}$. hep-th/0612304 (2006)

[9] Fonseca, P., Zamolodchikov, A.: Ward identities and integrable differential equations in the Ising field theory. hep-th/0309228 (2003)

[10] Fonseca, P., Zamolodchikov, A.: Ising field theory in a magnetic field: analytic properties of the free energy. J. Stat. Phys. 110(3-6), 527-590 (2003)

[11] 't Hoof, G.: A two-dimensional model for mesons. Nucl. Phys. B 75(3), 461-470 (1974)

[12] Jimbo, M., Miwa, T., Môri, Y., Sato, M.: Density matrix of an impenetrable bose gas and the fifth Painlevé transcendent. Physica D 1(1), 80-158 (1980)

[13] Kenzelmann, M., Batista, C.D., Chen, Y., Broholm, C., Reich, D.H., Park, S., Qiu, Y.: $S=\frac{1}{2}$ chain in a staggered field: high-energy boundspinon state and the effect of a discrete lattice. Phys. Rev. B 71(9), 094,411 (2005)

[14] McCoy, B.M., Wu, T.T.: Two dimensional Ising field theory in a magnetic field: breakup of the cut in the two-point function. Phys Rev. D 18(4), 1259-1267 (1978)

[15] Muskhelishvili, N.I.: Singular Integral Equations. Noordhoff International Publishing, Leyden (1977) 
[16] Onsager, L.: Crystal statistics. I. A two-dimensional Ising model with an order-disorder transition. Phys. Rev. 65(3-4), 117-149 (1944)

[17] Pikin, S.A., Tsukernik, V.M.: Thermodynamics of linear spin chains in a transverse magnetic field. Zh. Eksp. Teor. Fiz. 50(5), 1377-1380 (1966) [Sov. Phys. JETP 23, 914 (1966)]

[18] Rutkevich, S.B.: Large- $n$ excitations in the ferromagnetic Ising field theory in a weak magnetic field. Phys. Rev. Lett. 95(25), 25,0601 (2005)

[19] Rutkevich, S.B., Yashin, A.N., Kopeliovich, A.I., Yanovsky, A.V.: Bound-spinon excitations in the ferromagnetic Ising spin chain in a skew magnetic field. Nonlinear Phenomena in Complex Systems, in press.

[20] Sachdev, S.: Quantum Phase Transitions. Cambridge University Press, Cambridge (1999)

[21] Vekua, I.N.: On the Prandtl integro-differential equation. Prikladnaja Matematika i Mehanika 9, 143-150 (1945)

[22] Yurov, V.P., Zamolodchikov, Al.B.: Truncated-fermionic space approach to the critical $2 \mathrm{~d}$ Ising model with magnetic field. Int. J. Mod. Phys. A 6(25), 4557-4578 (1991)

[23] Zamolodchikov, A.B.: Integrals of motion and $S$-matrix of the (scaled) $T=T_{c}$ Ising model with magnetic field. Int. J. Mod. Phys. A 4(16), 4235-4248 (1989)

[24] Ziman, J.M.: Principles of the Theory of Solids. University Press, Cambridge (1972) 